\title{
Germline $B R C A 2$ mutation is associated with greater progression-free survival in korean women with advanced high-grade serous ovarian cancer
}

\author{
Ji Geun Yoo ${ }^{1}$, Hae Nam Lee ${ }^{2}$, Sung Jong Lee ${ }^{3}$, Jin Hwi Kim ${ }^{4}$, Yong Seok Lee ${ }^{5}$, Ahwon Lee ${ }^{6}$, \\ Soo Young Hur ${ }^{3}$, Keun Ho Lee ${ }^{3}$ \\ ${ }^{1}$ Department of Obstetrics and Gynecology, Daejeon St Mary's Hospital, College of Medicine, The Catholic University of Korea, \\ Seoul, Republic of Korea \\ ${ }^{2}$ Department of Obstetrics and Gynecology, Bucheon St. Mary's Hospital, College of Medicine, The Catholic University of Korea, \\ Seoul, Republic of Korea \\ ${ }^{3}$ Department of Obstetrics and Gynecology, Seoul St. Mary's Hospital, College of Medicine, The Catholic University of Korea, \\ Seoul, Republic of Korea \\ ${ }^{4}$ Department of Obstetrics and Gynecology, Uijeongbu St. Mary Hospital, College of Medicine, The Catholic University of Korea, \\ Seoul, Republic of Korea \\ ${ }^{5}$ Department of Obstetrics and Gynecology, Eunpyeong St. Mary's Hospital, College of Medicine, The Catholic University of Korea, \\ Seoul, Republic of Korea \\ ${ }^{6}$ Department of Hospital Pathology, Seoul St. Mary's Hospital, College of Medicine, The Catholic University of Korea, Seoul, \\ Republic of Korea
}

\begin{abstract}
Summary
Purpose: In this study, the effect of a germline BRCA1/2 mutation on survival outcomes in Korean patients with advanced high-grade serous ovarian cancer was investigated. Methods: This multicenter retrospective study involved six Korean institutions that included 63 patients with International Federation of Gynecology and Obstetrics stages IIIC or IV high-grade serous ovarian cancer, diagnosed surgically between January 2010 and December 2015, and who had undergone a $B R C A$ mutation test during their follow-up period. Median progression-free survival and 5-year overall survival were measured and compared according to BRCA1/2 mutation status using the Kaplan-Meier method. Results: Of 63 patients, 28 (44.4\%) patients with germline BRCA1/2 mutations were identified. Nineteen $(30.2 \%)$ patients were identified with a germline $B R C A 1$ mutation, and nine (14.3\%) patients with a germline $B R C A 2$ mutation. The median progression-free survival of patients with wild-type $B R C A$, or a $B R C A 1$ or $B R C A 2$ mutation was 15,17 and 37 months, respectively. Wild-type $B R C A$ and germline $B R C A 2$ mutation groups showed a significant difference in progression-free survival $(p=0.009)$; however, a significant difference was not found between wild-type $B R C A$ and germline $B R C A 1$ mutation groups $(p=0.262)$. Five-year overall survival rates of patients with wild-type $B R C A$, or a $B R C A 1$ or $B R C A 2$ mutation were $65.9 \%, 67.5 \%$ and $87.5 \%$, respectively; significant differences was not found between the three groups. Conclusion: In Patients with advanced high grade serous ovarian cancer, germline $B R C A 2$ mutation group showed a significantly longer median progression-free survival compared to wild-type $B R C A$ group. Five-year overall survival rates were not significantly different among the three groups.
\end{abstract}

TRN: KCT0005463

Date of registration: 2020-10-12

Key words: BRCA mutation; Korea; Multicenter study; Ovarian neoplasm; Survival analysis.

\section{Introduction}

The $B R C A$ gene is a tumor suppressor gene related to hereditary ovarian cancer. Hereditary ovarian cancer accounts for over $10 \%$ of all ovarian cancers, with $90 \%$ reported to be correlated with a BRCA gene mutation [1]. The prevalence of a $B R C A$ mutation was shown to be about $12-14 \%$ among patients with invasive ovarian cancer with racial variations [2-4]. Patients with a $B R C A 1$ gene mutation have a $44 \%$ lifetime risk of ovarian cancer, whereas those with a $B R C A 2$ gene mutation show about a $17 \%$ risk [5].

A $B R C A 1 / 2$ mutation in patients with ovarian cancer is associated with high-grade serous ovarian carcinoma (HGSOC) [6], a more advanced stage at diagnosis [6, 7], and an increased platinum-sensitivity [8]. A number of studies have sought to clarify the association between $B R C A \mathrm{mu}-$ tation status and survival outcome. However, most studies have included patients with various histological types and stages of ovarian cancer. Since ovarian cancer is character-
Eur. J. Gynaecol. Oncol. - ISSN: 0392-2936 XLI, n. 5, 2020

doi: $10.31083 /$ j.ejgo.2020.05.5255
This is an open access article under the CC BY-NC 4.0 license (https://creativecommons.org/licenses/by-nc/4.0/). 


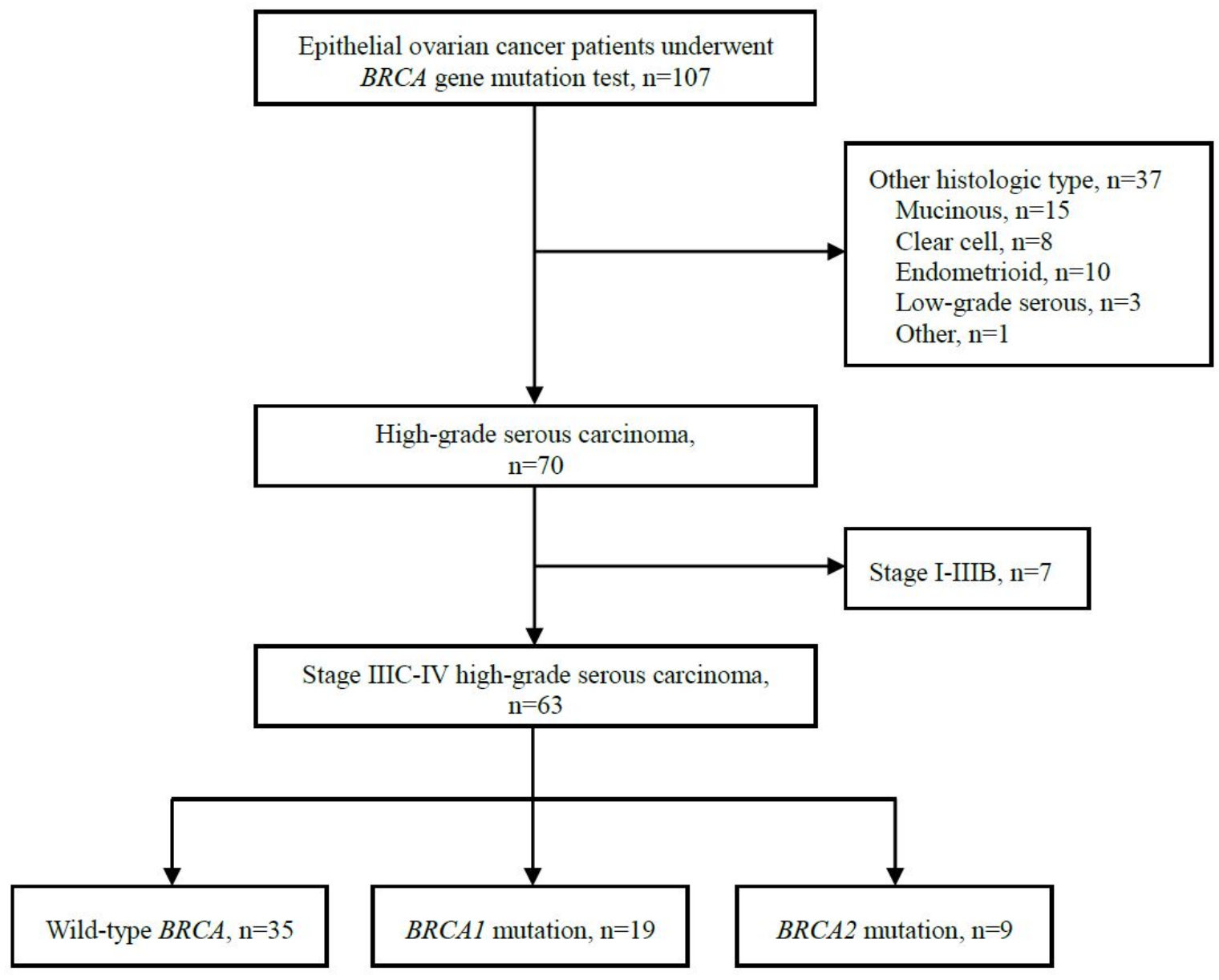

Figure 1. - Patient selection diagram.

ized by heterogeneity in morphology, prognosis, pathogenesis and molecular pathology [9], survival outcomes need to be studied in more specific patient populations to better reflect the characteristics of ovarian cancer with a $B R C A$ mutation.

This multicenter retrospective study aimed to investigate survival outcomes according to germline $B R C A$ mutations among Korean patients with advanced HGSOC.

\section{Methods}

This study was approved by The Catholic University of Korea Catholic Medical Center Institutional Review Board (XC16RIMI0004K). We retrospectively reviewed the medical records of 107 patients who underwent testing for a $B R C A$ gene mutation after being diagnosed with epithelial ovarian cancer between January 2010 and December 2015 in hospitals affiliated with the Catholic University of Korea, including Seoul St. Mary's Hospital, Yeouido St. Mary's Hospital, Bucheon St. Mary's Hospital, Uijeongbu St. Mary's Hospital, St. Vincent's Hospital, and Daejeon St. Mary's Hospital. Of these patients, 63 diagnosed with stage IIIC or IV HGSOC were included. Figure 1 shows the patient selection process. Fifty-two (82.5\%) patients un- derwent primary debulking surgery, and all had undergone at least six cycles of carboplatin-paclitaxel chemotherapy. The remaining 11 patients were histologically diagnosed with HGSOC by ascites cytology or biopsy, and had undergone three cycles of neoadjuvant chemotherapy with carboplatin-paclitaxel, followed by an interval debulking operation and at least three cycles of chemotherapy. We obtained EDTA-anticoagulated whole blood samples from patients during their chemotherapy cycle or a follow-up period; samples were sent to genetic laboratories to be analyzed for BRCA1/2 mutation status. St. Vincent's Hospital proceeded with genetic testing at the Samkwang Medical Laboratories, Seoul, Korea, and Daejeon St. Mary’s Hospital undertook testing at Green Cross Genome Research Center, Yongin-si, Korea. The remaining four hospitals sent their samples to the Catholic Genetic Laboratory of Seoul St. Mary's Hospital, Seoul, Korea. All laboratories evaluated $B R C A 1 / 2$ mutation status by the Sanger sequencing method.

The medical and familial history of patients was collected at the first visit and on admission for surgery. Patients were divided into two groups: those who had a personal history of breast or ovarian cancer or one among their 
Table 1. - Clinical characteristics of patients $(n=63)$

\begin{tabular}{|c|c|c|c|c|}
\hline Variables & Wild-type BRCA $(\mathrm{n}=35)$ & BRCA1 mutation $(\mathrm{n}=19)$ & BRCA2 mutation $(\mathrm{n}=9)$ & $p$-value \\
\hline Mean age, yr & $55.7[39-74]$ & $52.1[38-70]$ & $57.2[45-71]$ & 0.351 \\
\hline Mean body mass index, $\mathrm{kg} / \mathrm{m}^{2}$ & $23.5[18.3-33.9]$ & $23.5[19.3-28.9]$ & $23.9[20.5-30.4]$ & 0.993 \\
\hline Menopaused & $21(60.0)$ & $11(57.9)$ & $8(88.9)$ & 0.368 \\
\hline \multicolumn{5}{|l|}{ Family history positive group } \\
\hline Yes & $5(14.3)$ & $8(42.1)$ & $5(55.6)$ & 0.015 \\
\hline No & $30(85.7)$ & $11(57.9)$ & $4(44.4)$ & \\
\hline \multicolumn{5}{|l|}{ FIGO Stage } \\
\hline IIIC & $33(94.3)$ & $17(89.5)$ & $8(88.9)$ & 0.879 \\
\hline IV & $2(5.7)$ & $2(10.5)$ & $1(11.1)$ & \\
\hline NAC and IDS & $8(22.9)$ & $3(15.8)$ & 0 & 0.307 \\
\hline Success of $\mathrm{R} 0$ resection & $29(82.9)$ & $13(68.4)$ & $7(77.8)$ & 0.609 \\
\hline $\begin{array}{l}\text { Mean CA-125, mg/dL } \\
\text { at diagnosis }\end{array}$ & $1781.2[115.0-9481]$ & $1898.3[107.4-5500]$ & $783[42.6-2621]$ & 0.556 \\
\hline at the end of initial treatment & $10.4[1.2-38.5]$ & $15.6[3.4-96.8]$ & $9.2[2.6-27.4]$ & 0.595 \\
\hline Recurrence & $31(88.6)$ & $15(78.9)$ & $5(55.6)$ & 0.13 \\
\hline Platinum resistant recurrence & $5(14.3)$ & $4(21.1)$ & 0 & 0.53 \\
\hline Median follow-up period, mo. & 49 [13-92] & 61 [17-99] & $46[38-91]$ & 0.261 \\
\hline
\end{tabular}

Data are number (\%) or value [range]. NAC: Neoadjuvant chemotherapy, IDS: interval debulking surgery, FIGO: International Federation of Gynecology and Obstetrics, CA: cancer antigen.

first-degree relatives were defined as part of the "family history positive group"; other patients were defined as the "sporadic group".

During follow-up periods, the cancer antigen (CA)-125 titer and clinical findings of patients were evaluated every 3 months for the first 2 years, every 6 months for the following 3 years, and then annually. Abdominopelvic computed tomography (CT) was performed when clinical evidence of a recurrence or an elevation of the CA-125 titer was observed, otherwise CT was performed every 6 months for the first 5 years. Based on Response Evaluation Criteria In Solid Tumors (RECIST) and PET Response Criteria In Solid Tumors (PERCIST) [10, 11], the cancer recurrence date was defined as the date of detecting a recurrence of cancer. Progression free survival (PFS) was defined as the period from the start of treatment to the cancer recurrence date, and overall survival (OS) was the period from the start of treatment to death from any cause.

Statistical analysis was performed using IBM SPSS Version 20 for Windows. PFS and OS were analyzed by the Kaplan-Meier method, and significance was confirmed by log-rank test.

\section{Results}

Table 1 shows the clinical characteristics of patients. Of the 63 patients, a germline $B R C A 1$ mutation was found in $19(30.2 \%)$ patients, while a germline $B R C A 2$ mutation was found in nine $(14.3 \%)$ patients. None of the patients had concurrent germline $B R C A 1$ and $B R C A 2$ mutations. Unclassified variations of the $B R C A 1$ gene were found in seven patients, and variations of $B R C A 2$ were found in 14 patients.

A germline $B R C A$ mutation was found in 13 (72.2\%) of the 18 patients of the family history positive group. Eight (44.4\%) patients had a germline $B R C A 1$ mutation, and five (27.8\%) patients had a germline $B R C A 2$ mutation. In the sporadic group, $11(24.4 \%)$ of 45 patients had a germline $B R C A 1$ mutation, and four $(8.9 \%)$ patients had a germline $B R C A 2$ mutation. Univariate analysis for each group divided by $B R C A$ mutation status showed a significant difference in family or personal history of breast and ovarian cancers, but significant differences in mean age, body mass index, menopausal status, successful no residual resection rate, and follow-up period were not noted.

The PFS and OS were analyzed according to $B R C A$ gene mutation status. Kaplan-meier survival curves showing the PFS (A) and the OS (B) are presented in Figure 2. The median follow-up period was 49 months. A significantly longer median PFS was noted for the germline $B R C A 1 / 2$ mutation group compared to the wild-type $B R C A$ group (28 months [95\% confidence interval (CI) 7.3-48.7] vs. 15 months [12.1-17.9], $p=0.025)$. The median PFS of the germline BRCA1 mutation group was 17 months (7.0$27.0)$, and did not show a statistically significant difference compared to that of the wild-type BRCA group ( $p=0.262$ ), while the $B R C A 2$ mutation group showed a significantly longer median PFS (37 months [34.1-40.0], $p=0.009$ ).

The 5-year OS was $65.9 \%, 67.5 \%$ and $87.5 \%$ for wildtype $B R C A$, and $B R C A 1$ or $B R C A 2$ mutation groups, respectively, without significant differences observed between groups.

\section{Discussion}

Ovarian cancer is newly diagnosed in about 240,000 people and is the cause of death in about 150,000 people 


\begin{tabular}{ll}
\hline 十 $B R C A$ wild type & - $B R C A 1 / 2$ mutation \\
- $B R C A 1$ mutation & - $B R C A 2$ mutation \\
\hline
\end{tabular}

A

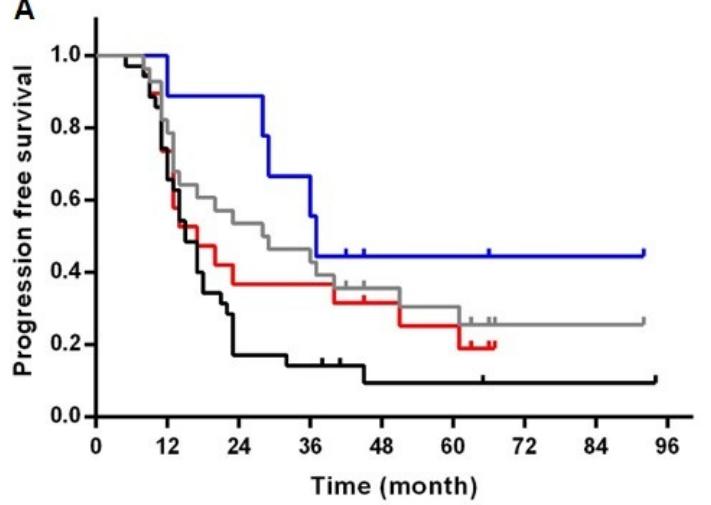

B

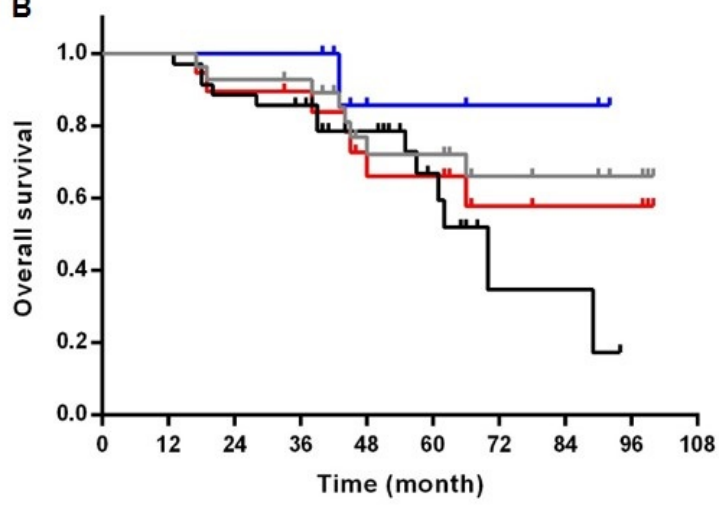

Figure 2. - Kaplan-Meier survival plots of progression-free survival and overall survival for each group. A: Progression-free survival. The log-rank p values for wild-type $B R C A$ vs. $B R C A 1 / 2, B R C A 1$ and $B R C A 2$ mutations are $0.026,0.262$ and 0.009 , respectively. For $B R C A 1$ mutation $v s$. BRCA2 mutation, $p=0.174$. B: Overall survival. The log-rank $p$ values for wild-type $B R C A v s . B R C A 1 / 2, B R C A 1$ and $B R C A 2$ mutations are $0.160,0.437$ and 0.059 , respectively. For $B R C A 1$ mutation $v s$. BRCA2 mutation, $p=0.271$.

worldwide every year [12]. In Korea, about 2,400 patients are newly diagnosed with ovarian cancer yearly, and that number is gradually increasing [13]. At the time of diagnosis, about $75 \%$ of patients have advanced stage cancer [14].

About $90 \%$ of hereditary ovarian cancers, comprising over $10 \%$ of overall epithelial ovarian cancer cases, are associated with a germline $B R C A$ gene mutation [1]. $B R C A$ gene mutations have an autosomal dominant inheritance pattern and occur in many other cancers such as breast, stomach, colon, and pancreas. Therefore, relatives of patients with a germline $B R C A$ mutation should consider genetic testing and counseling [15], which would enable the early diagnosis and prevention of ovarian cancer through oral contraceptives or a risk-reducing salpingooophorectomy [16, 17]. Furthermore, targeted therapy, such as poly ADP ribose polymerase inhibitors, have become a valuable additional treatment option [18-21].

Cancers associated with $B R C A$ gene mutations are sensitive to chemotherapy $[1,8]$. However, the suggestion that patients with $B R C A$ mutations have better outcomes is still controversial. Previous studies attempted to determine the correlation between such mutations and overall survival. Although several studies reported better overall survival in patients with $B R C A$ gene mutations [22, 23], other studies did not demonstrate the same trend [24-26].

Ovarian cancer is a heterogeneous disease, consisting of various histologic types and stages. Each shows a different chemosensitivity and oncologic outcome [9]. Previous studies were often analyzed, including various histologic types and stages. In this study, patients with high-grade serous histology and FIGO stages IIIC-IV were included to better represent the characteristics of $B R C A$ mutant ovarian cancer. Based on these criteria, of 107 patients with nonselective epithelial ovarian cancer, $41 B R C A$ mutation negative patients were excluded, whereas only three $B R C A$ mutation positive patients were excluded.

For the same reason, we analyzed the survival outcomes of patients with germline $B R C A 1$ and $B R C A 2$ mutations separately. Many studies have analyzed $B R C A 1$ and $B R C A 2$ mutations combined as one, but these genes have different functions in the homologous recombination pathway, resulting in differences in the lifetime risk of $B R C A$ mutation-associated cancers and clinical outcomes [27]. As a result of this study, patients who have a germline $B R C A 2$ mutation showed a marked and statistically significant median PFS compared to those without a $B R C A$ mutation, whereas $B R C A 1$ mutation groups showed no statistical significance. These results are consistent with a previous study in a comprehensive analysis of The Cancer Genome Atlas data [28]. In this study, we show that combining $B R C A 1$ and BRCA2 mutations into one when undertaking survival analysis is likely to result in overestimating the survival benefit of the $B R C A 1$ mutation.

Five-year OS rates showed no significant differences between the three groups. To date, we could obtain information about the five-year OS rates in $65.1 \%$ of all patients. Deaths related to disease progression occurred in 21 patients $(33.3 \%)$, and, moreover, only one death occurred in the $B R C A 2$ mutation group. Further data on overall survival in these patients will be updated in future studies.

To our knowledge, this is the first multicenter study conducted in Asia to analyze survival outcomes according to 
$B R C A$ mutation status. The prevalence and types of $B R C A$ mutations in ovarian cancer differ according to ethnicity. Also, Asian ovarian cancer patients show different clinicopathologic characteristics, responses to drugs, and survival outcomes to those of westerners [29, 30]. However, only a few studies of the implication of $B R C A$ mutations on survival outcome in patients with ovarian cancer have been conducted in Asia [8, 31]. To provide patients with more accurate survival information and to apply this to treatment, we suggest large-scale studies need to be performed.

This study has several limitations. The number of patients included in this study was small, with only nine patients with a $B R C A 2$ mutation investigated. Larger studies are needed to accurately determine the oncological outcomes of a specific $B R C A$ mutation status. In addition, this study evaluated germline $B R C A$ mutation status by Sanger sequencing. However, in Korea, next-generation sequencing has been certified by the Ministry of Food and Drug Safety since 2016, while previously treated patients underwent $B R C A$ mutation testing with Sanger sequencing. It is also known that about $50 \%$ of HGSOC is related to homologous recombination deficiency [32]. The clinical significance of genetic lesions other than germline $B R C A \mathrm{mu}-$ tations, such as somatic BRCA mutations, RAD51, ATM, $B R I P 1, C H E K 2$ mutations, and epigenetic modifications, has not been examined in this study.

In conclusion, we have demonstrated the effect of specific $B R C A$ mutations on the survival of patients with HGSOC in this study. Compared to the wild-type $B R C A$ group, patients with a germline $B R C A 2$ mutation showed prolonged PFS; however, those with a $B R C A 1$ mutation had a similar PFS to that of those with wild-type $B R C A$. An additional large-scale prospective study is needed to confirm these results, while the final overall survival data of this study is pending.

\section{Acknowledgments}

We would like to thank our peer reviewers and editors for their helpful comments and suggestions.

\section{Conflict of Interest}

The authors declare that they have no conflict of interest in this study.

Submitted: May 12, 2019

Accepted: October 30, 2019

Published: October 15, 2020

\section{References}

[1] Boyd J.: "Specific Keynote: Hereditary Ovarian Cancer: What We Know". Gynecol. Oncol., 2003, 88, S8-S10.

[2] Zhang S., Royer R., Li S., McLaughlin J.R., Rosen B., Risch H.A., et al.: "Frequencies of BRCA1 and BRCA2 mutations among 1,342 unselected patients with invasive ovarian cancer". Gynecol. Oncol., 2011, 121, 353-357.

[3] Lim M.C., Kang S., Seo S., Kong S., Lee B., Lee S., et al.: "BRCA1 and BRCA2 germline mutations in Korean ovarian cancer patients". J. Cancer Res. Clin. Oncol., 2009, 135, 1593-1599.
[4] Weiderpass E., Tyczynski J. E.: "Epidemiology of Patients with Ovarian Cancer with and Without a BRCA12 Mutation". Molecular Diagnosis \& Therapy, 2015, 19, 351-364.

[5] Kuchenbaecker K.B., Hopper J.L., Barnes D.R., Phillips K.A., Mooij T.M., Roos-Blom M.J. et al.: "Risks of Breast, Ovarian, and Contralateral Breast Cancer for BRCA1 and BRCA2 Mutation Carriers". JAMA., 2017, 317, 2402-2416.

[6] Alsop K., Fereday S., Meldrum C., deFazio A., Emmanuel C., George J., et al.: "BRCA Mutation Frequency and Patterns of Treatment Response in BRCA Mutation-Positive Women With Ovarian Cancer: A Report From the Australian Ovarian Cancer Study Group". J. Clin. Oncol., 2012, 30, 2654-2663.

[7] Hirsh-Yechezkel G., Chetrit A., Lubin F., Friedman E., Peretz T. Gershoni R., et al.: "Population attributes affecting the prevalence of BRCA mutation carriers in epithelial ovarian cancer cases in israel". Gynecol. Oncol., 2003, 89, 494-498.

[8] Tan D.S.P., Rothermundt C., Thomas K., Bancroft E., Eeles R., Shanley S., et al.: "BRCAness" Syndrome in Ovarian Cancer: A Case-Control Study Describing the Clinical Features and Outcome of Patients With Epithelial Ovarian Cancer Associated WithBRCA1andBRCA2Mutations". J. Clin. Oncol., 2008, 26, 55305536.

[9] Meinhold-Heerlein I., Hauptmann S.: "The heterogeneity of ovarian cancer". Arch. Gynecol. Obstet., 2014, 289, 237-239.

[10] Eisenhauer E.A., Therasse P., Bogaerts J., Schwartz L.H., Sargent D., Ford R., et al.: "New response evaluation criteria in solid tumours: Revised RECIST guideline (version 1.1)". Eur. J. Cancer 2009, 45, 228-247.

[11] Wahl R.L., Jacene H., Kasamon Y. and Lodge M.A.: "From RECIST to PERCIST: Evolving Considerations for PET Response Criteria in Solid Tumors". J. Nucl. Med., 2009, 50, 122S-150S.

[12] Wild C.P.: "International Agency for Research on Cancer". Encyclopedia of Toxicology, 2014, 1067-1069.

[13] Jung K.W., Won Y.J., Oh C.M., Kong H.J., Lee D.H., Lee K.H.: "Ministry of Health and Welfare, Korea: Annual report of cancer registry programme in the Republic of Korea". Cancer Res. Treat., 2017, 49, 292.

[14] Jelovac D. and Armstrong D.K.: "Recent progress in the diagnosis and treatment of ovarian cancer". Ca. Cancer J. Clin., 2011, 61, 183-203.

[15] Brose M.S.: "Cancer Risk Estimates for BRCA1 Mutation Carriers Identified in a Risk Evaluation Program". Cancerspectrum Knowledge Environment, 2002, 94, 1365-1372.

[16] Moorman P.G., Havrilesky L.J., Gierisch J.M., Coeytaux R.R., Lowery W.J., Peragallo Urrutia R., et al.: "Oral Contraceptives and Risk of Ovarian Cancer and Breast Cancer Among High-Risk Women: A Systematic Review and Meta-Analysis". J. Clin. Oncol., 2013, 31, 4188-4198.

[17] Domchek S.M., Friebel T.M., Singer C.F., Evans D.G., Lynch H.T. Isaacs C. et al:: "Association of risk-reducing surgery in BRCA1 or BRCA2 mutation carriers with cancer risk and mortality". JAMA, 2010, 304, 967.

[18] Liu J.F., Matulonis U.A.: "What Is the Place of PARP Inhibitors in Ovarian Cancer Treatment?" Curr. Oncol. Rep., 2016, 18,

[19] Ledermann J., Harter P., Gourley C., Friedlander M., Vergote I., Rustin G., et al.: "Olaparib maintenance therapy in patients with platinum-sensitive relapsed serous ovarian cancer: a preplanned retrospective analysis of outcomes by BRCA status in a randomised phase 2 trial". the Lancet Oncology, 2014, 15, 852-861.

[20] Oza A.M., Cibula D., Benzaquen A.O., Poole C., Mathijssen R.H.J., Sonke G.S., et al.: "Olaparib combined with chemotherapy for recurrent platinum-sensitive ovarian cancer: a randomised phase 2 trial". the Lancet Oncology, 2015, 16, 87-97.

[21] Ledermann J., Harter P., Gourley C., Friedlander M., Vergote I., Rustin G., et al.: "Olaparib Maintenance Therapy in PlatinumSensitive Relapsed Ovarian Cancer". N. Engl. J. Med., 2012, 366, 1382-1392.

[22] Zhong Q., Peng H., Zhao X., Zhang L., Hwang W.: "Effects of BRCA1- and BRCA2-Related Mutations on Ovarian and Breast Cancer Survival: A Meta-analysis". Clin. Cancer Res., 2015, 21, 211-220.

[23] Bolton K.L., Chenevix-Trench G., Goh C., Sadetzki S., Ramus S.J., Karlan B.Y. et al:: "Association between BRCA1 and BRCA2 mutations and survival in women with invasive epithelial ovarian cancer". JAMA, 2012, 307, 382. 
[24] Kotsopoulos J., Rosen B., Fan I., Moody J., McLaughlin J.R., Risch H., et al.: "Ten-year survival after epithelial ovarian cancer is not associated with BRCA mutation status". Gynecol. Oncol., 2016, 140, 42-47.

[25] McLaughlin J.R., Rosen B., Moody J., Pal T., Fan I., Shaw P.A., et al.: "Long-Term Ovarian Cancer Survival Associated With Mutation in BRCA1 or BRCA2". JNCI: Journal of the National Cancer Institute, 2013, 105, 141-148.

[26] Candido-dos-Reis F.J., Song H., Goode E.L., Cunningham J.M., Fridley B.L., Larson M.C. et al.: "Germline mutation in BRCA1 or $B R C A 2$ and ten-year survival for women diagnosed with epithelial ovarian cancer". Clin. Cancer Res., 2015, 21, 652-657.

[27] Liu G., Yang D., Sun Y., Shmulevich I., Xue F., Sood A.K., et al.: "Differing clinical impact ofBRCA1 andBRCA2mutations in serous ovarian cancer". Pharmacogenomics, 2012, 13, 1523-1535.

[28] Yang D., Khan S., Sun Y., Hess K., Shmulevich I., Sood A.K., et al.: "Association of BRCA1 and BRCA2 Mutations With Survival, Chemotherapy Sensitivity, and Gene Mutator Phenotype in Patients With Ovarian Cancer". Jama, 2011, 306, 1557.

[29] Fuh K.C., Shin J.Y., Kapp D.S., Brooks R.A., Ueda S., Urban R.R., et al.: "Survival differences of Asian and Caucasian epithelial ovar- ian cancer patients in the United States". Gynecol. Oncol., 2015, 136, 491-497.

[30] du Bois A., Floquet A., Kim J., Rau J., del Campo J.M., Friedlander M., et al.: "Incorporation of Pazopanib in Maintenance Therapy of Ovarian Cancer". J. Clin. Oncol., 2014, 32, 3374-3382.

[31] Aida H., Takakuwa K., Nagata H., Tsuneki I., Takano M., Tsuji S. et al.: "Clinical features of ovarian cancer in Japanese women with germ-line mutations of BRCA1". Clin. Cancer. Res., 1998, 4, 235240 .

[32] The Cancer Genome Atlas Research Network: "Integrated genomic analyses of ovarian carcinoma". Nature, 2011, 474, 609-615.

Corresponding Author:

KEUN HO LEE, M.D., Ph.D.

Department of Obstetrics and Gynecology, Seoul St Mary's Hospital, College of Medicine, The Catholic University of Korea, 222 Banpodaero, Seocho-gu, Seoul, 06591 (Korea) e-mail: hohoho@catholic.ac.kr 